\title{
PROJECTIVITIES IN FINITE GROUPS
}

D. W. BARNES

(received 24 September 1963)

Chains of projectivities within the lattice $\mathscr{P}(G)$ of subnormal subgroups of a group $G$ have been considered by various authors, see for example Barnes [1] and Tamaschke [2]. The starting point of the investigations of which this paper is a report, was the theorem, basically due to Wielandt:

THEOREM 1. If $A / B$ is a non-abelian composition factor of a group $G$ with a finite composition series, then $A / B$ is not projective in $\mathscr{S}(G)$ to any other factor of the same composition series, and any chain of projections from $A / B$ to $A / B$ induces the identity automorphism in $A / B .^{1}$

This is proved by a "homotopy" argument in which the chain of projections is deformed into a chain with a very simple form. A similar argument proves

THEOREM 2. Let $A / B$ be a composition factor of prime order $p$ of a group $G$ with a finite composition series.

(i) If $A / B$ is projective to no other factor of the same composition series, then every chain of projections from $A / B$ to $A / B$ induces the identity automorphism.

(ii) If $A / B$ is projective to some other factor of the same composition series, then every automorphism of $A / B$ is induced by some chain of projections in $\mathscr{P}(G)$.

This leads us to consider for $A$ subnormal in $G$ (which in this paper is always assumed finite) and $B$ normal in $A$, the group $\pi(A / B, G)$ of automorphisms of $A / B$ induced by chains of projections in $\mathscr{S}(G)$. We ask the question: for given $A / B$, what subgroups of the automorphism group of $A / B$ can be $\pi(A / B, G)$ for some suitable overgroup $G$ ?

The theorems quoted above give a complete answer to this question for $A / B$ simple. By the same method, it can be proved that $\pi(A / B, G)$ is trivial unless for some prime $p, A / B$ has a normal subgroup of index $p$. For an elementary abelian quotient $A / B, \pi(A / B, G)$ is non-trivial only if $A / B$ is projective in $\mathscr{S}(G)$ to a subgroup of a larger elementary abelian

1 See Zassenhaus [4] p. 235 exercises 20 and 21. 
quotient, in which case $\pi(A / B, G)$ is the group of all automorphisms of $A / B$. The next case to consider is the case where $A / B$ is a cyclic $p$-group of order greater than $p$.

In part I, we develop a homotopy theory of finite groups in sufficient generality for application to the general problem. In part II, we solve the problem for cyclic quotients $A / B$ in abelian $p$-groups. We remark that this solves the problem for a cyclic quotient in any finite abelian group since the lattice of a finite abelian group is the direct product of the lattices of its Sylow subgroups. If we denote by $G_{p}$ the unique Sylow $p$-subgroup of the abelian group $G$, and if $A / B$ is a quotient of $G$, then $\pi(A / B, G)$ is the direct product of the groups $\pi\left((A / B)_{D}, G_{D}\right)$.

\section{PART I. HOMOTOPY THEORY OF FINITE GROUPS}

\section{Projectivities}

A quotient in a group $G$ is a factor group $A / B$ where $A$ is a subgroup of $G$ and $B$ is a normal subgroup of $A$. Suppose $L$ is some sublattice of the lattice of all subgroups of $G$. The quotient ${ }^{2} A / B$ is called admissible with respect to $L$ if $A, B \in L$. The lattice $L$ is supposed fixed, and by "quotient" we shall mean "quotient admissible with respect to $L$ ". Suppose $A / B$, $C / D$ are (admissible) quotients and $A=B \cup C, D=B \cap C$. Then the

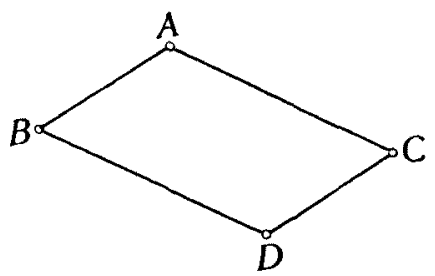

Fig. 1.

map $a B \rightarrow(a B) \cap C$ for all $a \in A$ of the cosets of $B$ in $A$ onto the cosets of $D$ in $C$ is an isomorphism. Such an isomorphism is called a projection down, its inverse is called a projection up.

A chain $c$ of projections is a sequence $\phi_{1}, \phi_{2}, \cdots, \phi_{n}$ of projections such that $\phi_{i}$ is a map from the image of $\phi_{i-1}(i=2,3, \cdots, n)$. Since the projection

$$
\phi_{i}: A_{i-1} / B_{i-1} \rightarrow A_{i} / B_{i}=\left(A_{i-1} / B_{i-1}\right) \phi_{i}
$$

is uniquely determined by the intervals $A_{i-1} / B_{i-1}$ and $A_{i} / B_{i}$, we write

2 Notice that not every quotient of $L$ in the lattice sense is a quotient in the sense of this paper. 


$$
c=\left(A_{0} / B_{0} \rightarrow A_{1} / B_{1} \rightarrow \cdots \rightarrow A_{n} / B_{n}\right) .
$$

$c$ clearly defines the isomorphism $\phi=\phi_{1} \phi_{2} \cdots \phi_{n}$ of $A_{0} / B_{0}$ onto $A_{n} / B_{n}$. Such an isomorphism $\phi$ is called a projectivity (in $L$ ). If

$$
d=\left(A_{n} / B_{n} \rightarrow A_{n+1} / B_{n+1} \rightarrow \cdots \rightarrow A_{n+m} / B_{n+m}\right)
$$

is a chain of projections giving the projectivity $\psi$ of $A_{n} / B_{n}$ onto $A_{n+m} / B_{n+m}$, then we define $c d$ to be the chain $c d=\left(A_{0} / B_{0} \rightarrow A_{1} / B_{1} \rightarrow \cdots \rightarrow A_{n} / B_{n} \rightarrow A_{n+1} / B_{n+1} \rightarrow \cdots \rightarrow A_{n+m} / B_{n+m}\right)$. Clearly $c d$ gives the projectivity $\phi \psi$ of $A_{0} / B_{0}$ onto $A_{n+m} / B_{n+m}$. We define $r^{-1}$ to be the chain

$$
c^{-1}=\left(A_{n} / B_{n} \rightarrow \cdots \rightarrow A_{1} / B_{1} \rightarrow A_{0} / B_{0}\right) .
$$

$c^{-1}$ clearly gives the projectivity $\phi^{-1}$.

\section{The projectivity group $\pi(\Lambda)$}

If $c$ is a chain starting and finishing at the same quotient $A / B$, we call $c$ a loop. $c$ then gives an automorphism $\alpha(c)$ of $A / B$. It is convenient to define $\alpha(c)$ to be the identity automorphism for $c=(A / B)$, the trivial loop consisting of the empty set of projections. Clearly the set of all automorphisms $\alpha(c)$ of $A / B$ given by loops $c$ starting and finishing at $A / B$ is a group which we denote by $\pi(A / B)$. Two quotients $A / B, C / D$ are called projective (in $L$ ) if there exists a chain $d$ of projections

$$
d=\left(A / B \rightarrow A_{1} / B_{1} \rightarrow \cdots \rightarrow A_{n} / B_{n}=C / D\right) .
$$

If $A / B$ and $C / D$ are projective, then $\pi(A / B)$ and $\pi(C / D)$ are isomorphic. Thus $\pi(A / B)$ depends (up to isomorphism) only on the class $A$ of projective quotients to which $A / B$ belongs. We therefore denote the (isomorphism class of the) group by $\pi(\Lambda)$.

\section{The fundamental group $F(\Lambda)$}

If in the chain $c=\left(A_{0} / B_{0} \rightarrow A_{1} / B_{1} \rightarrow \cdots \rightarrow A_{n} / B_{n}\right)$, we have $A_{r+2} / B_{r+2}=A_{r} / B_{r}$ for some $r$, then the projection $A_{r+1} / B_{r+1} \rightarrow A_{r+2} / B_{r+2}$ is the inverse of the projection $A_{r} / B_{r} \rightarrow A_{r+1} / B_{r+1}$. If

$$
c^{\prime}=\left(A_{0} / B_{0} \rightarrow \cdots \rightarrow A_{r} / B_{r}=A_{r+2} / B_{r+2} \rightarrow A_{r+3} / B_{r+3} \rightarrow \cdots \rightarrow A_{n} / B_{n}\right)
$$

is the chain formed from $c$ by omitting the projections

$$
A_{r} / B_{r} \rightarrow A_{r+1} / B_{r+1} \rightarrow A_{r+2} / B_{r+2}=A_{r} / B_{r},
$$

then $c$ and $c^{\prime}$ give the same projectivity. We say that $c$ and $c^{\prime}$ can be obtained from one another by a transformation of type (1). 
If there exist projections $A_{1} / B_{1} \rightarrow A_{2} / B_{2}, \quad A_{1} / B_{1} \rightarrow A_{3} / B_{3}$ and $A_{2} / B_{2} \rightarrow A_{3} / B_{3}$, then $A_{1} / B_{1} \rightarrow A_{2} / B_{2} \rightarrow A_{3} / B_{3}$ and $A_{1} / B_{1} \rightarrow A_{3} / B_{3}$ give the same projectivity. This follows from the fact that, for some permutation $u, v$, $w$ of $1,2,3$, we have $A_{u} \geqq A_{v} \geqq A_{w}, B_{u} \geqq B_{v} \geqq B_{w}$ and fer the coset $a B_{u}$ of $B_{u}$ in $A_{u}$,

$$
\left(\left(a B_{u}\right) \cap B_{v}\right) \cap B_{w}=\left(a B_{u}\right) \cap B_{w} .
$$

If the chain $c^{\prime}$ is obtained from $c=\left(A_{1} / B_{1} \rightarrow \cdots \rightarrow A_{n} / B_{n}\right)$ by replacing $A_{i+1} / B_{i+1} \rightarrow A_{i+2} / B_{i+2} \rightarrow A_{i+3} / B_{i+3}$ by $A_{i+1} / B_{i+1} \rightarrow A_{i+3} / B_{i-3}$ where $A_{i+u} \geqq A_{i+v} \geqq A_{i+w}, B_{i+u} \geqq B_{i+v} \geqq B_{i+v}$ for some permuliati is $i$, $v$, $w$ of $1,2,3$, then the transformation of replacing $c$ by $c^{\prime}$ or if replicing $c^{\prime}$ by $c$ is called a transformation of type (2).

We say that the two loops $c, c^{\prime}$ starting at $A / B$ are ${ }^{\prime}$.umotopic if $c^{\prime}$ can be obtained from $c$ by a succession of transformations $i$, rypes (1) and (2), such that the chain obtained after each successive transformation is again a loop starting at $A / B$. The classes of homotopic loops starting at $A / B$ clearly form a group $F(A / B)$ which we call the fundamental group at $A / B$. As in ordinary homotopy theory, this group depends (up to isomorphism) only on the class $A$ of projective quotients to which $A / B$ belongs, not on the actual starting point $A / B$ of the loops, and we therefore denote the fundamental group by $F(\Lambda)$. Clearly $c \rightarrow \alpha(c)$ defines a homomorphism $\alpha: F(\Lambda) \rightarrow \pi(\Lambda)$. Thus $\pi(\Lambda)$ can be determined if we can calculate $\alpha(c)$ for a representative loop $c$ of each element in some generating set of $F(\Lambda)$.

We have called this group $F(\Lambda)$ the fundamental group of the class $\Lambda$. This is consistent with the terminology of topology if we make $A$ a two dimensional complex in the following manner. The vertices of the complex $A$ are the quotients $A / B \in A$ and the edges are the projections $A / B \rightarrow C / D$ in $A$. This defines the one-skeleton of the complex. Every triangle $A|B \rightarrow C| D \rightarrow E / F \rightarrow A \mid B$ in $A$ is spanned by a surface. These surfaces spanning a triangle are the faces of the complex $A$. The fundamental group of this complex is precisely the group $F(\Lambda) .^{3}$

\section{The homology group $H(\Lambda)$}

We introduce a further two types of transformations of chains. If $c=u a b v$ where $a, b$ are loops starting at $U / V, u$ is a chain ending at $U / V$ and $v$ is a chain starting at $U / V$ for some $U / V \in A$, and if $c^{\prime}=u b a v$, then we say that $c^{\prime}$ is obtained from $c$ by a transformation of type (3). The equivalence classes of loops starting at some fixed $A / B$ outainable from one another by transformations of types (1), i2: . A (3) fcrm the abelia:

- For the homotopy theory of complexes, seu selfert and Threlfas $\lfloor 3\rfloor$ p. $162-100$. 
group $F(A / B) / F^{\prime}(A / B)$ which is the factor group of $F(A / B)$ by its commutator subgroup.

If $c=\left(A_{1} / B_{1} \rightarrow A_{2} / B_{2} \rightarrow \cdots \rightarrow A_{n} / B_{n} \rightarrow A_{1} / B_{1}\right)$, and $c^{\prime}=\left(A_{r} / B_{r}\right.$ $\left.\rightarrow A_{r+1} / B_{r+1} \rightarrow \cdots \rightarrow A_{n} / B_{n} \rightarrow A_{1} / B_{1} \rightarrow \cdots \rightarrow A_{r} / B_{r}\right)$ consists of the same projections as $c$ taken in the same cyclic order, then we say $c^{\prime}$ is obtained from $c$ by a transformation of type (4).

If $c, c^{\prime}$ are loops in $A$ and $c^{\prime}$ can be obtained from $c$ by a succession of transformations of types (1), (2), (3) and (4), we say that $c, c^{\prime}$ are homologous loops. The classes of homologous loops form an abelian group $H(\Lambda)$ which we call the homology group of $A$.

If $u$ is a chain from $A / B$ to $C / D$, then the map taking the loop $c$ starting at $A / B$ to the loop $w^{-1} C u$ starting at $C / D$ gives an isomorphism of $F(A / B)$ onto $F(C / D)$. This isomorphism is not in general unique but depends on $u$ to the extent of an inner automorphism. Consequently the induced map of $F(A / B) / F^{\prime}(A / B)$ onto $F(C / D) / F^{\prime}(C / D)$ is independent of $u$. The element of $F(A \mid B) / F^{\prime}(A \mid B)$ with the loop $c$ as representative is precisely the set of loops starting at $A / B$ and homologous to $c$. The element of $F(C / D) / F^{\prime}(C / D)$ corresponding to this under the map induced by $c \rightarrow u^{-1} c u$ is the set of loops starting at $C / D$ and homologous to $c .^{4}$ Thus if we identify corresponding elements of the different $F(A \mid B) / F^{\prime}(A / B)$, the group whose elements are these classes of identified elements is the group $H(\Lambda) . H(\Lambda) \simeq F(\Lambda) / F^{\prime}(\Lambda)$ is the one-dimensional homology group of the two-dimensional complex $A$.

If the group $\pi(\Lambda)$ is abelian, then the map $c \rightarrow \alpha(c)$ induces a homomorphism of $H(\Lambda)$ onto $\pi(\Lambda)$. In this case, $\pi(\Lambda)$ can be determined if we can find the $\alpha(c)$ given by the elements of a generating set for $H(\Lambda)$.

\section{The restricted complex}

The elements of $A$ are naturally partially ordered, $A / B \geqq C / D$ if $A \geqq C, B \geqq D$ and $A=B \cup C, D=B \cap C$, i.e. $A \mid B \geqq C / D$ if there is a projection down from $A / B$ to $C / D$. The maximal elements of $A$ in this partial ordering we call the peaks of $A$, the minimal elements we call the pits.

If $A / B \rightarrow C / D$ is a projection up, and $C / D \rightarrow E / F$ is a projection down, and if $C^{\prime} \mid D^{\prime}$ is a peak, $C^{\prime} \mid D^{\prime} \geqq C / D$, then $A / B \rightarrow C / D \rightarrow E / F$ is homotopic with fixed end-points to $A / B \rightarrow C^{\prime} \mid D^{\prime} \rightarrow E / F$. Thus if $A / B$ is a peak or pit, then in every homotopy class in $F(A / B)$ there is a loop $A / B=A_{0} / B_{0} \rightarrow A_{1} / B_{1} \rightarrow \cdots \rightarrow A_{n} / B_{n} \rightarrow A / B$ in which the $A_{i} / B_{i}$ are alternately peaks and pits. It is convenient to study these loops, considering only those homotopies in which all intermediate loops obtained by the successive transformations have this same form. In this, we use

4 See Seifert and Threlfall [3] p. 174-176. 
transformations of type (1) only. This leads us to consider the complex $A^{*}$ whose vertices are the peaks and pits of $\Lambda$ and whose edges are the projections between these peaks and pits. The faces of $A$ were introduced to allow transformations of type (2). Since these cannot occur between loops of the type we are considering, we do not define faces of $\Lambda^{*}$. We call $\Lambda^{*}$ the restricted complex of $\Lambda$. Its fundamental group $F\left(\Lambda^{*}\right)$, which we denote by $F^{*}=F^{*}(\Lambda)$, we call the restricted fundamental group of $\Lambda$. The homology group $H\left(\Lambda^{*}\right)=H^{*}=H^{*}(\Lambda)$ of $\Lambda^{*}$ we call the restricted homology group of $\boldsymbol{A}$.

Each restricted homotopy class of loops is contained in a homotopy class, and each homotopy class with peak or pit as starting point contains at least one restricted class. The map in which each element of $F^{*}$ maps into the element of $F$ in which it is contained, is clearly a homomorphism of $F^{*}$ onto $F$. Similarly there is a homomorphism of $H^{*}$ onto $H$.

Since $\Lambda^{*}$ is a one-dimensional complex, $F^{*}$ is a free group and $H^{*}$ is free abelian ${ }^{5}$. This, together with the fact that the vertices and edges of $\Lambda^{*}$ are more easily enumerated than those of $\Lambda$ makes $F^{*}$ and $H^{*}$ more convenient tools than $F$ and $H$ for the determination of $\pi(\Lambda)$. $F^{*}$ and $H^{*}$ are not necessarily groups of simpler structure than $F$ and $H$, for example, if $L$ is the lattice of all subgroups of the elementary abelian group of order 8 and the vertices of $\Lambda$ are all the quotients of order 2 , then $F(\Lambda)$ is trivial but $F^{*}(\Lambda)$ is the free group on 15 generators.

\section{Covering spaces}

For each of the complexes $\Lambda, \Lambda^{*}$ we can define in a natural way a covering space whose monodromie group is $\pi(\Lambda)^{6}$.

Let $K$ be either of the complexes $\Lambda, A^{*}$. Each vertex $V$ of $K$ is a quotient $A / B$ of the group $G$. The points of the covering space $\bar{K}$ which lie over $V$ are the elements of $A / B$. If $V_{1}=A_{1} / B_{1}$ is joined to $V_{2}=A_{2} / B_{2}$ in $K$ by an edge, then in $\widetilde{K}$ each element $a_{1} \in A_{1} / B_{1}$ is joined by an edge to the element $a_{2} \in A_{2} / B_{2}$ to which it maps under the projection $A_{1} / B_{1} \rightarrow A_{2} / B_{2}$. Every triangle in $\tilde{K}$ is spanned by a face. The points $a_{1}, a_{2}, a_{3} \in \tilde{K}$ can be vertices of a face only if the points $V_{1}, V_{2}, V_{3}$ of $K$ over which they lie are vertices of a face of $K$. Conversely, if $V_{1}, V_{2}, V_{3}$ are vertices of a face of $K$ and if $a_{1}$ lies over $V_{1}, a_{2}, a_{3}$ the images of $a_{1}$ under the projections $V_{1} \rightarrow V_{2}$ and $V_{1} \rightarrow V_{3}$, then $a_{3}$ is the image of $a_{2}$ under the projection $V_{2} \rightarrow V_{3}$ and $a_{1}, a_{2}, a_{3}$ are the vertices of a face of $\tilde{K}$. If $n$ is the order of the quotients in $A$, then over each vertex of $K$ lie $n$ points of $\tilde{K}, n$ edges of $\tilde{K}$ lie over

see Seifert and Threlfall [3] p. 165-168.

- For the theory of covering spaces, see Seifert and Threlfall [3], chapter 8, p. 181-203. The monodromie group is discussed in $\$ 58$, p. 198-203. 
each edge of $K$ and $n$ faces of $\tilde{K}$ lie over each face of $K$. By taking suitable coordinate systems in the faces and edges of $K$ and $\tilde{K}$ and mapping each point of $\tilde{K}$ to the point in the corresponding face or edge of $K$ and with the same coordinates, we can clearly define a map of $\tilde{K}$ onto $K$ making $\tilde{K}$ a covering space for $K$.

The theory of covering spaces is usually developed under the assumption that the covering space is connected. If the quotients in $A$ are not trivial, then $\widetilde{K}$ cannot be connected since any projection preserves the order of an element, and in particular, maps the identity element to the identity element. We can however apply the theory of connected covering spaces to the connected components of $\tilde{K}$.

Take any vertex $V$ of $K$ and let $a_{1}, \cdots, a_{n}$ be the points of $K$ lying over $V$. For any loop $c$ in $K$ starting at $V$, there is a uniquely defined path $p_{i}$ starting at $a_{i}$ and lying over $c$. The map which takes $a_{i}$ into the point at which the path $p_{i}$ ends is a permutation of the points $a_{i}$. The monodromie group of $\widetilde{K}$ is the group of all such permutations. Since the end-point of $p_{i}$ is $a_{i} \alpha(c)$, the monodromie group is precisely the group $\pi(\Lambda)$. Since $\tilde{K}$ is not connected, the monodromie group of $K$ is not transitive.

A connected covering space $\tilde{W}$ of the complex $W$ is called regular if, for every loop $l$ in $W$, the paths $p_{\imath}$ in $\tilde{W}$ lying over $l$ are either all closed or all open. Since every transitive abelian permutation group is regular, every component of $\tilde{K}$ is regular if $\pi(\Lambda)$ is abelian, in particular, if the quotients $A / B \in A$ are cyclic. If on the other hand, $A$ is a class of elementary abelian quotients of order $p^{n}(n \geqq 2)$ in $\mathscr{S}(G)$ and $\pi(\Lambda)$ is not trivial, then $\pi(\Lambda)$ is the general linear group $G L(n, p)$ and is transitive on the nonidentity elements of any quotient in $\Lambda$. A connected covering space is regular if and only if its multiplicity is equal to the order of its monodromie group. In this example, $\pi(\Lambda)$ is the monodromie group of the non-identity component of $A$. Since the multiplicity of the component is $p^{n}-1$ which is not equal to $|\pi(\Lambda)|=\left(p^{n}-1\right)\left(p^{n}-p\right) \cdots\left(p^{n}-p^{n-1}\right)$, it is not a regular covering space.

\section{Lengths}

Suppose $c$ is a loop in $\Lambda$ or $\Lambda^{*}$. We define the length of $c$ to be the number of projections in $c$. Suppose $T$ is one of the groups $F, F^{*}, H, H^{*}$ and that $t$ is an element of $T$. (In the case of $F$, we suppose the starting point of the loops to be some fixed peak or pit.) Then $t$ is a class of loops. We define the length $l(t)$ of $t$ to be the length of the shortest loop in $t$. Since the shortest loop in $t$ must consist of projections alternately up and down, $l(t)$ is an even integer. $t$ is called reducible if there exist $t_{1} t_{2}, \cdots, t_{r} \in T$ such that $t=t_{1} t_{2} \cdots t_{r}$ and $l\left(t_{i}\right)<l(t)$ for all $i$. Otherwise $t$ is called irreducible. 
We denote by $T^{(n)}$ the subgroup of $T$ generated by all elements of $T$ of length less than or equal to $2 n$. The length $l(T)$ of $T$ is the length of its longest irreducible element. If $l(T)=2 n$, then $T=T^{(n)} \neq T^{(n-1)}$.

If $T$ is $F$ or $F^{*}$, then an element of $T$ is a class of loops with fixed endpoint. Although the group $T$ (up to isomorphism) is independent of that fixed end-point, $l(T)$ may depend on the end-point. For example, suppose $G=\langle a, b\rangle, a^{4}=b^{2}=a b a^{-1} b^{-1}=1$, and $L$ the lattice of all subgroups of $G$.

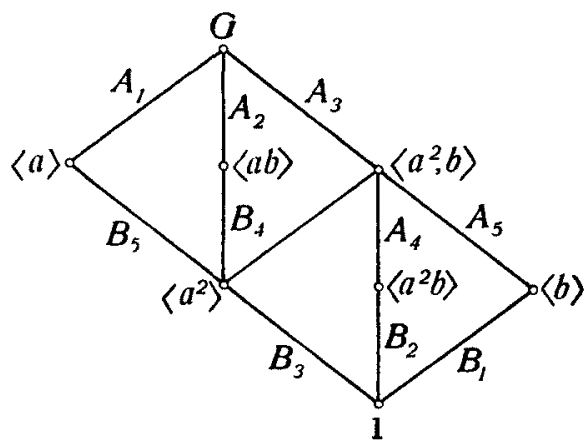

Fig. 2.

We consider the quotients of order 2. The peaks of $A$ are $A_{1}=G \mid\langle a\rangle$, $A_{2}=G\left|\langle a b\rangle, A_{3}=G\right|\left\langle a^{2}, b\right\rangle, A_{4}=\left\langle a^{2}, b\right\rangle\left|\left\langle a^{2} b\right\rangle, A_{5}=\left\langle a^{2}, b\right\rangle\right|\langle b\rangle$ and the pits are $B_{1}=\langle b\rangle, B_{2}=\left\langle a^{2} b\right\rangle, B_{3}=\left\langle a^{2}\right\rangle, B_{4}=\langle a b\rangle\left|\left\langle a^{2}\right\rangle, B_{5}=\langle a\rangle\right|\left\langle a^{2}\right\rangle$ as marked on the lattice diagram. $A^{*}$ is the complex

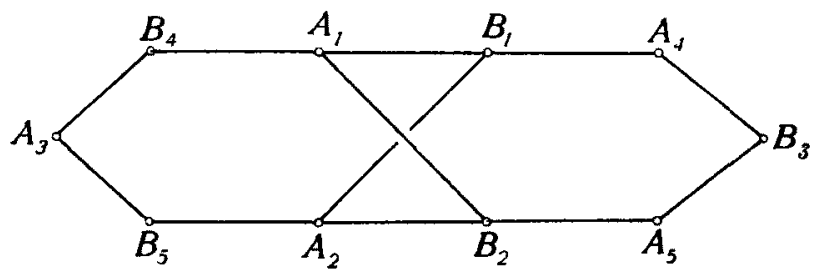

Fig. 3.

$F^{*}\left(A_{1}\right)$ is generated by the irreducible loops $\left(A_{1} \rightarrow B_{1} \rightarrow A_{2} \rightarrow B_{2} \rightarrow A_{1}\right)$,

and

$$
\left(A_{1} \rightarrow B_{4} \rightarrow A_{3} \rightarrow B_{5} \rightarrow A_{2} \rightarrow B_{1} \rightarrow A_{1}\right)
$$

$$
\left(A_{1} \rightarrow B_{1} \rightarrow A_{4} \rightarrow B_{3} \rightarrow A_{5} \rightarrow B_{2} \rightarrow A_{1}\right) \text {. }
$$

Thus $l\left(F^{*}\left(A_{1}\right)\right)=6 . F^{*}\left(A_{3}\right)$ is generated by the irreducible loops 
and

$$
\begin{aligned}
& \left(A_{3} \rightarrow B_{4} \rightarrow A_{1} \rightarrow B_{2} \rightarrow A_{2} \rightarrow B_{5} \rightarrow A_{3}\right), \\
& \left(A_{3} \rightarrow B_{4} \rightarrow A_{1} \rightarrow B_{1} \rightarrow A_{2} \rightarrow B_{5} \rightarrow A_{3}\right)
\end{aligned}
$$

$$
\left(A_{3} \rightarrow B_{4} \rightarrow A_{1} \rightarrow B_{1} \rightarrow A_{4} \rightarrow B_{3} \rightarrow A_{5} \rightarrow B_{2} \rightarrow A_{2} \rightarrow B_{5} \rightarrow A_{3}\right) .
$$

Thus $l\left(F^{*}\left(A_{3}\right)\right)=10$. Thus to define $F^{(n)}, F^{*(n)}, l(F)$ or $l\left(F^{*}\right)$, it is necessary to specify the point at which the loops start. This difficulty does not arise with $H$ and $H^{*}$. Trivially, $l(H) \leqq l(F)$ and $l\left(H^{*}\right) \leqq l\left(F^{*}\right)$.

LEMMA 7.1. $H / H^{(2)} \simeq H^{*} / H^{*(2)}$.

Proof. Let $c=\left(A_{1} / B_{1} \rightarrow A_{2} / B_{2} \rightarrow \cdots \rightarrow A_{n} / B_{n} \rightarrow A_{1} / B_{1}\right)$ be a representative loop of an element of $H$. We may choose $c$ such that the projections are alternately up and down. If $A_{i} / B_{i} \rightarrow A_{i+1} / B_{i+1}$ is a projection up, take $A_{i+1}^{\prime} / B_{i+1}^{\prime}$ any peak such that $A_{i+1}^{\prime} / B_{i+1}^{\prime} \geqq A_{i+1} / B_{i+1}$, and $A_{i}^{\prime} / B_{i}^{\prime}$ any pit such that $A_{i}^{\prime} / B_{i}^{\prime} \leqq A_{i} / B_{i}$. Put

$$
c^{\prime}=\left(A_{1}^{\prime} / B_{1}^{\prime} \rightarrow A_{2}^{\prime} / B_{2}^{\prime} \rightarrow \cdots \rightarrow A_{n}^{\prime} / B_{n}^{\prime} \rightarrow A_{1}^{\prime} / B_{1}^{\prime}\right) .
$$

Then $c^{\prime}$ is a loop in $A^{*}$ and gives an element of $H^{*} / H^{*(2)}$ which is completely determined by $c$. This clearly defines a homomorphism of $H$ onto $H^{*} / H^{*(2)}$, and $H^{(2)}$ is clearly contained in the kernel. Since the loops $c$ and $c^{\prime}$ are homologous, $c^{\prime}$ is a representative of an element of $H^{*(2)}$ only if $c$ is a representative of an element of $H^{(2)}$. Hence $H / H^{(2)} \simeq H^{*} / H^{*(2)}$.

\section{PART II. GYCLIC QUOTIENTS IN ABELIAN p-GROUPS}

We now restrict ourselves to the case where $G$ is a finite abelian $p$ group, $L$ is the lattice of all subgroups of $G$ and $A$ is a class of projective cyclic quotients. The group $G$ is a direct product of cyclic groups $\left\langle u_{1}\right\rangle, \cdots,\left\langle u_{k}\right\rangle$ of orders $p^{\lambda_{1}}, \cdots, p^{\lambda_{k}}$. We may suppose the numbering of the $u_{i}$ chosen so that $\lambda_{1} \geqq \lambda_{2} \geqq \cdots \geqq \lambda_{k}$, in which case we say that $G$ is of type $\left(\lambda_{1}, \cdots, \lambda_{k}\right)$. Let $p^{\lambda}$ be the order of the quotients in $\Lambda$. We shall determine the number of classes $A$ of cyclic quotients of order $p^{\lambda}$ in $G$, show that the groups $F(\Lambda), F^{*}(\Lambda), H(\Lambda), H^{*}(\Lambda)$ and $\pi(\Lambda)$ depend only on $p, \lambda, \lambda_{1}, \cdots, \lambda_{k}$, not on the actual class $\Lambda$ chosen, and determine $\pi(\Lambda)$ in terms of these.

\section{The classes of projective cyclic quotients}

LEMMA 8.1. Let $G$ be any $p$-group and $C$ a cyclic group of order $p^{\lambda}$. Then every cyclic quotient of order $p^{\lambda}$ in $G \times C$ is projective to $C$.

Proof. Let $A / B$ be such a guotient. Then $A C / B$ is abelian of type 
$(\lambda, r), r \leqq \lambda$, and therefore $(G \cap A C) / G \cap B$, being isomorphic to a subgroup of $A C / B$, is abelian of type $\left(r_{1}, r_{2}\right), \lambda \geqq r_{1} \geqq r_{2}$. Since $A C=(A C \cap G) C$, we may take $G=A C \cap G$ without loss of generality. Since trivially $(G \cap B) C / G \cap B$ is projective to $C$, we may further suppose $G \cap B=1$. Thus we need only consider the case $G$ abelian of type $\left(r_{1}, r_{2}\right), r_{i} \leqq \lambda$. Then $G \times C$ has exponent $p^{\lambda}$. Take any coset representative $a$ of a generator of $A \mid B$. Then $\langle a\rangle$ is a cyclic subgroup of $G \times C$ and $A \mid B \rightarrow\langle a\rangle$ is a projection. $\langle a\rangle C$ is an abelian group of type $(\lambda, r), r \leqq \lambda$. There exists $D \leqq\langle a\rangle C$, $|D|=p^{r}$ such that $D \cap\langle a\rangle=D \cap C=1$. Then $\langle a\rangle \rightarrow\langle a\rangle C \mid D \rightarrow C$ is a chain of projections.

THEOREM $3 .{ }^{7}$ Let $G$ be an abelian p-group of type $\left(\lambda_{1}, \cdots, \lambda_{k}\right)$.

(a) If $\lambda_{2} \geqq \lambda$, then $G$ has only one class of projective cyclic quotients of order $p^{\lambda}$.

(b) If $\lambda_{1}=\lambda+r, \lambda_{2}<\lambda$, then $G$ has precisely $r+1$ classes of projective cyclic quotients of order $p^{\lambda}$. If $G=U \times V$ where $U$ is cyclic of order $p^{\lambda_{1}}$, then these classes are the sets of all cyclic quotients of order $p^{\lambda}$ of $\left(U^{p^{i}} \times V\right) / U^{p^{x+\lambda}}$ for $i=0,1, \cdots, r$.

Proof. (a) $G$ is the direct product of cyclic groups $U_{i}$ of orders $p^{\lambda_{i}}$. Suppose $A / B$ is a cyclic quotient of order $p^{\lambda}$, and let $a$ be a coset representative of a generator of $A \mid B$. Then $A / B \rightarrow\langle a\rangle /\langle a\rangle \cap B$ is a projection. At least one of $\langle a\rangle \cap U_{1},\langle a\rangle \cap U_{2}$ is trivial and therefore at least one of $\langle a\rangle U_{1}^{p_{1}^{\lambda_{1}-\lambda}},\langle a\rangle U_{2}^{p_{2}^{\lambda_{2}-\lambda}}$ is a direct product. By lemma $8.1,\langle a\rangle \mid\langle a\rangle \cap B$ is projective to $U_{2}^{p_{1}^{\lambda_{1}}-\lambda}$ for $i=1$ or $i=2$. But $U_{1}^{p^{\lambda_{1}-\lambda}}$ is projective to $U_{2}^{p^{\lambda_{2}}-\lambda}$. Hence all cyclic quotients of order $p^{\lambda}$ are projective to $U_{1}^{\lambda_{2}-\lambda}$

(b) Suppose $A / B$ is a cyclic quotient of order $p^{\lambda}$. For some $i, U^{p^{i}} \times V \geqq A$ but $U^{p^{i+1}} \times V \geq A$. Then $A=\left\langle u^{v^{i}} v, V\right\rangle$ for some $v \in V$, where $\langle u\rangle=U$. $B \geqq A^{p \lambda}$ and therefore $u^{p^{1+2}} \in B$. Therefore $A / B$ is a quotient of $\left(U^{p^{t}} \times V\right) / U^{p^{1+2}}$. By lemma 8.1, all cyclic quotients of $\left(U^{p^{i}} \times V\right) / U^{p^{t+\lambda}}$ of order $p^{\lambda}$ are projective to $U^{p^{t}} / U^{p^{t+\lambda}}$.

We complete the proof by showing that $A_{1} / B_{1}, A_{2} / B_{2}$ cyclic quotients of order $p^{\lambda}, A_{1} / B_{1}$ a quotient of $\left(U^{p^{i}} \times V\right) / U^{p^{1+2}}, A_{2} / B_{2}$ a quotient of $\left(U^{p^{\prime}} \times V\right) / U^{p^{j+2}}$ and $A_{1} / B_{1} \rightarrow A_{2} / B_{2}$ a projection implies $i=j$. If we denote by $\exp G$ the exponent of the group $G$, then we have $\exp A_{1}=p^{\lambda_{1}-i}>$ $\exp B_{1}$ and $\exp A_{2}=p^{\lambda_{1}-3}$. If $A_{1} / B_{1} \rightarrow A_{2} / B_{2}$ is a projection down, then $A_{1}=B_{1} A_{2}$ and $\exp A_{1}=\max \left\{\exp A_{2}, \exp B_{1}\right\}$. Therefore $\exp A_{1}=\exp A_{2}$ and $i=j$.

The groups $\left(U^{p^{4}} \times V\right) / U^{p^{1+\lambda}} i=0,1, \cdots, r$ are isomorphic, hence we have:

7 The classes of projective quotients of order $p$ in any $p$-group have also been determined. See Barnes [1], theorem 2.1, Tamaschke [2], theorem 3.3 . 
CoROLlary 8.2. If $G$ is a finite abelian p-group and $A$ a class of projective cyclic quotients of order $p^{\lambda}$ in $G$, then the groups $F(\Lambda), F^{*}(\Lambda), H(\Lambda), H^{*}(\Lambda)$ and $\pi(\Lambda)$ depend only on $G$ and $\lambda$, not on the particular class $\Lambda$.

\section{Classification of the peaks and pits}

Lemma 9.1. $A / B$ is a pit in $G$ if and only if $A$ is cyclic. Dually, $A / B$ is a peak if and only if $G / B$ is cyclic.

Proof. Trivially, if $A$ is cyclic, then $A / B$ is a pit. If $A$ is not cyclic, take a coset representative $a$ of a generator of $A / B$. Then $A|B \rightarrow\langle a\rangle|\langle a\rangle \cap B$ is a projection down.

LEMMA 9.2. Suppose $A / B, C / B$ are pits, $A^{p^{\lambda-1}}=C^{p \lambda-1}$ and $E / F$ is a peak, $E / F \rightarrow A / B$ a projection. Then there is a projection $E / F \rightarrow C / B$.

PRoOF. $F \geqq C^{p^{\lambda-1}}, \quad F \geqq B=C^{p^{\lambda}}$ and therefore $F \cap C=B$. $C / B \rightarrow C \cup F / F$ is a projection. Therefore $|C \cup F: F|=p^{\lambda}$. But $G / F$ is cyclic and therefore $C \cup F=E$.

LEMma 9.3. Let $c$ be a loop in $A^{*}, A / B, C / B$ pits such that $A^{p \lambda-1}=C^{p \lambda-1}$. Let $c^{\prime}$ be a loop obtained from $c$ by replacing $A / B$ by $C / B$ at some (not necessarily all) occurrences of $A / B$ in $c$. Let $(c),\left(c^{\prime}\right)$ be the homology classes (in $\Lambda^{*}$ ) of $c, c^{\prime}$. Then

$$
(c) H^{*(2)}=\left(c^{\prime}\right) H^{*(2)}
$$

Proof. We consider only the case in which one change is made.

$$
\begin{aligned}
c & =\left(\cdots \rightarrow E_{1} / F_{1} \rightarrow A / B \rightarrow E_{2} / F_{2} \rightarrow \cdots\right) \\
c^{\prime} & =\left(\cdots \rightarrow E_{1} / F_{1} \rightarrow C / B \rightarrow E_{2} / F_{2} \rightarrow \cdots\right) .
\end{aligned}
$$

$c^{\prime}$ is a chain of projections by the previous lemma. Put

$$
h=\left(E_{1} / F_{1} \rightarrow A / B \rightarrow E_{2} / F_{2} \rightarrow C / B \rightarrow E_{1} / F_{1}\right) .
$$

Then $(c)=\left(c^{\prime}\right)(h)$ and $(h) \in H^{*(2)}$.

We define an equivalence relation on the pits, putting $A / B \sim C / D$ if $A^{p^{\lambda-1}}=C^{p^{\lambda-1}}$. Then the above two lemmas state that, in any loop representing an element of $H^{*}$, replacing a pit $A / B$ by an equivalent pit gives a loop representing an element of $H^{*}$ in the same coset of $H^{*(2)}$. Thus working in $H^{*} / H^{*(2)}$, we may replace pits by equivalent pits.

Dually, we say that the peaks $A|B, A| D$ are equivalent if $A^{D} B=A^{D} D$. Then working in $H^{*} / H^{*(2)}$ we may replace any peak by an equivalent peak.

We now give the classes of equivalent peaks and pits homogeneous coordinates, and find a condition for the existence of a projection from a peak to a pit in terms of the coordinates of the equivalence classes to 
which they belong. We call a class of equivalent pits a point and a class of equivalent peaks we call a line. We say that the point $P$ lies on the line $l$ if for some $A / B \in P$ and $C / D \in l$ there is a projection $A / B \rightarrow C / D$. By the lemmas above, if $P$ lies on $l$, then for every $A / B \in P$ and $C / D \in l$, there is a projection $A / B \rightarrow C / D$. The "plane" consisting of these points and lines with this incidence relation, we denote by $\Pi$. It is not a projective plane.

Suppose $G=\left\langle u_{1}, \cdots, u_{k}\right\rangle, \quad u_{2}^{p^{\lambda_{i}}}=1, \quad \lambda_{1} \geqq \lambda_{2} \geqq \cdots \geqq \lambda_{r} \geqq \lambda$, $\lambda>\lambda_{r+1} \geqq \lambda_{r+2} \geqq \cdots \geqq \lambda_{k}$. If $A / B \in P$, then $A$ is cyclic and therefore $A=\left\langle u_{1}^{x_{1}} u_{2}^{x_{2}} \cdots u_{k}^{x_{k}}\right\rangle$ for some $x_{1}, \cdots, x_{k} . P$ is completely determined by $A^{p^{\lambda-1}}=\left\langle v_{1}^{x_{1}} v_{2}^{x_{2}} \cdots v_{r}^{x_{r}}\right\rangle$ where $v_{2}=u_{1}^{p \lambda-1}$. We take the row vector $\boldsymbol{x}=\left(x_{1}, \cdots, x_{r}\right)$ as a coordinate vector for $P$. The $x_{i}$ are integers mod $p^{\lambda_{i}-\lambda+1}$. If $t$ is an integer $\neq 0 \bmod p$, then $t \boldsymbol{x}=\left(t x_{1}, t x_{2}, \cdots, t x_{r}\right)$ is again a coordinate vector for $P$ and all coordinate vectors for $P$ can be obtained in this way from any one. The points $P=\{A \mid B\}$ of $I$ are in one-to-one correspondence with the non-trivial cyclic subgroups $H=A^{p^{\lambda-1}}$ of $\Omega_{\lambda-1}(G)=\left\{x^{p^{\lambda-1}} \mid x \in G\right\}$.

If $l=\{C / D\}$ is a line of $\Pi$, then $K=C^{p} D$ is the same for all peaks $C / D \in l$, and $l$ is determined by $K$.

$$
K \geqq \Omega_{\lambda-1}(G)=\left\{x \mid x \in G, x^{p^{\lambda-1}}=1\right\},
$$

and $G / K$ is a non-trivial cyclic group. Since the lattice of all subgroups of $G$ is self-dual, the lines of $\Pi$ are in one-to-one correspondence with the subgroups $K<G, K \geqq \Omega_{\lambda-1}$ such that $G / K$ is cyclic, such $K$ being the dual of the non-trivial cyclic subgroups $H$ of $\sigma_{\lambda-1}(G)$. Since for every such $K, G / K$ is isomorphic to a non-trivial subgroup of the additive group $Z_{p^{\mu}}$ of integers $\bmod p^{\mu}$, where $\mu=\lambda_{1}-\lambda+1$, the lines of $\Pi$ are in one-to-one correspondence with the kernels $K / \Omega_{\lambda-1}$ of non-zero homomorphisms of $G / \Omega_{\lambda-1}$ into $Z_{p^{\mu}}$. If we put $w_{i}=u_{\imath} \Omega_{\lambda-1}$, then $G / \Omega_{\lambda-1}=\left\{w_{1}^{x_{1}} \cdots w_{r}^{x_{r}}\right\}$ where the $x_{i}$ are integers $\bmod p^{\lambda_{i}-\lambda+1}$. Every homomorphism of $G / \Omega_{\lambda-1}$ into $Z_{p^{\mu}}$ has the form $w_{1}^{x_{1}} \cdots w_{r}^{x_{r}} \rightarrow \sum_{i=1}^{r} l_{i} x_{i} p^{\lambda_{1}-\lambda_{i}} \bmod p^{\mu}$, where $l_{i}$ are integers $\bmod p^{\lambda_{r}-\lambda+1}$. We define the scalar product of the row vectors $\boldsymbol{l}=\left(l_{1}, \cdots, l_{r}\right), \boldsymbol{x}=\left(x_{1}, \cdots, x_{r}\right)$ where $l_{i}, x_{2}$ are integers $\bmod p^{\lambda_{i}-\lambda+1}$ to be the residue class $\bmod p^{\mu}$ given by

$$
\boldsymbol{l} \cdot \boldsymbol{x}=\sum_{i=1}^{r} l_{i} x_{i} p^{\lambda_{1}-\lambda_{i}} \quad\left(\bmod p^{\mu}\right)
$$

Every homomorphism of $G / \Omega_{\lambda-1}$ into $Z_{p^{\mu}}$ then has the form $w_{1}^{x_{1}} \cdots w_{r}^{x_{r}} \rightarrow \boldsymbol{l} \cdot \mathbf{x}$ and has kernel

$$
K / \Omega_{\lambda-1}=\left\{w_{1}^{x_{1}} \cdots w_{r}^{x_{r}} \mid \boldsymbol{l} \cdot \boldsymbol{x}=0\right\}
$$

$G / K$ is non-trivial if and only if $\boldsymbol{l} \neq \mathbf{0}$. We take the row vector $\boldsymbol{l}$ as a coor- 
dinate vector for the line $l$ corresponding to $K$. If $t \neq 0$ mod $p$, then $t \boldsymbol{l}$ is also a coordinate vector for $l$ and all coordinate vectors for $l$ may be obtained in this way.

Lemma 9.4. The point $P$ with coordinate vector $x$ lies on the line $l$ with coordinate vector $l$ if and only if

$$
\left\{\begin{array}{r}
\boldsymbol{l} \cdot \boldsymbol{x} \neq 0 \\
p \boldsymbol{l} \cdot \boldsymbol{x}=0
\end{array} \quad\left(\bmod p^{\mu}\right)\right.
$$

Proof. Let $A / B$ be a pit in $P, C / D$ a peak in $l$ and let $K=C^{p} D$. Clearly, if there exists a projection $A / B \rightarrow C / D$, then $A \leqq K, A^{\nu} \leqq K$. Conversely, if $A \leqq K, A^{p} \leqq K$ then $A \cap K=A^{p}$ and $A K / K \simeq A \mid A^{p}$. But

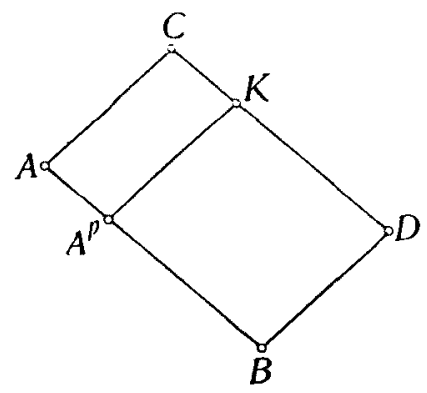

Fig. 4

$G / K$ is cyclic and has only one subgroup $C / K$ of order $p$. Hence $A K=C . G / D$ is cyclic and $A D \$ K$, so $A D>K$. Therefore $A D=C$. $A D / D \simeq A / A \cap D$ and $A$ has only one subgroup $B$ of index $p^{\lambda}$. Therefore $A \cap D=B$ and there is a projection $A / B \rightarrow C / D$.

$$
\begin{aligned}
K & =\left\{u_{1}^{\theta_{1}} \cdots u_{r}^{\theta_{r}} u_{r+1}^{\theta_{r+1}} \cdots u_{k}^{\theta_{k}} \mid \boldsymbol{l} \cdot\left(\theta_{1}, \cdots, \theta_{r}\right)=0\right\} . \\
A & =\left\langle u_{1}^{x_{1}} \cdots u_{r}^{x_{r}} u_{r+1}^{x_{r+1}} \cdots u_{k}^{x_{k}}\right\rangle \\
A^{p} & =\left\langle u_{1}^{p x_{1}} \cdots u_{k}^{p x_{k}}\right\rangle .
\end{aligned}
$$

Therefore $A \leqq K$ if and only if $l \cdot x=0$, and $A^{D} \leqq K$ if and only if $p \boldsymbol{l} \cdot \boldsymbol{x}=\mathbf{0}$. Therefore $\boldsymbol{l} \cdot \boldsymbol{x} \neq 0, p \boldsymbol{l} \cdot \boldsymbol{x}=0$ is a necessary and sufficient condition for $A \$ K, A^{p} \leqq K$ and hence for $P \in l$.

\section{The length of $H$}

Lemma 10.1. Suppose $\boldsymbol{u}, \boldsymbol{v}$ are vectors such that $\boldsymbol{u} \cdot \boldsymbol{x}=0$ implies $\boldsymbol{v} \cdot \boldsymbol{x}=\mathbf{0}$. Then $v=\alpha u$ for some integer $\alpha$.

Proof. Let $V$ be the additive group of the row vectors $x$. With each row vector $\boldsymbol{u}$, we have associated the homomorphism $\boldsymbol{u}: V \rightarrow Z_{\mathrm{p} \mu}$ given 


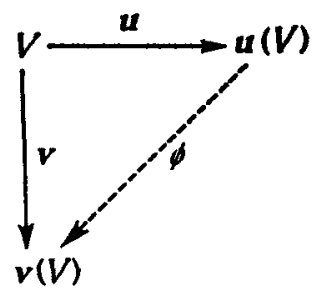

Fig. 6.

by $\boldsymbol{u}(\boldsymbol{x})=\boldsymbol{u} \cdot \boldsymbol{x}$. By hypothesis, ker $\boldsymbol{v} \geqq$ ker $\boldsymbol{u}$. Therefore there exists a homomorphism $\phi: u(V) \rightarrow v(V)$ such that $v=\phi u$. But $Z_{p \mu}$ is cyclic and its subgroups form a chain. Thus $\operatorname{ker} v \geqq \operatorname{ker} u$ implies im $v \leqq \operatorname{im} u$. If $z_{0}$ generates im $u$, then $\phi\left(z_{0}\right)=\alpha z_{0}$ for some integer $\alpha$. Since $\phi$ is a homomorphism, $\phi(z)=\alpha z$ for all $z \in \operatorname{im} u$. That is

$$
\begin{aligned}
v \cdot x & =\alpha(u \cdot x) \\
& =(\alpha u) \cdot x
\end{aligned}
$$

for all $x \in V$. Therefore $v=\alpha u$.

LEMMA 10.2. A necessary and sufficient condition for there to be a line of $\Pi$ through the points $P_{1}, P_{2}$ with coordinate vectors $a, b$ is that for all integers $\alpha, \beta$,

$$
\boldsymbol{a} \neq \alpha p \boldsymbol{b}, \quad \boldsymbol{b} \neq \beta p \boldsymbol{a},
$$

that is, $P_{1}, P_{2}$ are distinct collinear points if and only if $\theta a+\phi b=0$ implies $\theta \equiv \phi \equiv 0 \bmod p$.

Proof. Suppose that $a=\alpha p b$ for some integer $\alpha$. For any line with coordinates $\boldsymbol{l}$ passing through $P_{2}, p \boldsymbol{b} \cdot \boldsymbol{l}=0$ and therefore $\boldsymbol{a} \cdot \boldsymbol{l}=0$. Therefore no line through $P_{2}$ passes through $P_{1}$.

Conversely, suppose $a \neq \alpha p b, b \neq \beta p a$. We have to find $l$ such that

$$
\begin{array}{ll}
\boldsymbol{l} \cdot \boldsymbol{a} \neq 0 & \boldsymbol{p l} \cdot \boldsymbol{a}=0 \\
\boldsymbol{l} \cdot \boldsymbol{b} \neq 0 & \boldsymbol{p l} \cdot \boldsymbol{b}=\mathbf{0} .
\end{array}
$$

By lemma 10.1, there exists $x$ such that $p a \cdot x=0, b \cdot x \neq 0$. By replacing $\boldsymbol{x}$ by a suitable multiple, we can find $\boldsymbol{x}$ satisfying also $p \boldsymbol{b} \cdot \boldsymbol{x}=0$. If in addition, we have $a \cdot x \neq 0$, we take $l=x$. Similarly there exists $y$ such that $p a \cdot y=0, p b \cdot y=0$ and $a \cdot y \neq 0$. If also $b \cdot y \neq 0$, then we can take $\boldsymbol{l}=\boldsymbol{y}$. If both $\boldsymbol{a} \cdot \boldsymbol{x}=\mathbf{0}$ and $\boldsymbol{b} \cdot \boldsymbol{y}=0$, then we take $\boldsymbol{l}=\boldsymbol{x}+\boldsymbol{y}$.

Any loop $c=\left(A_{1} / B_{1} \rightarrow C_{1} / D_{1} \rightarrow A_{2} / B_{2} \rightarrow \cdots \rightarrow A_{1} / B_{1}\right)$ in $\Lambda^{*}$ gives a loop $\bar{c}=\left(P_{1} \rightarrow l_{2} \stackrel{l_{2}}{\rightarrow} \cdots \stackrel{l_{n}}{\rightarrow} P_{1}\right)$ in $\Pi$, where $P_{i}$ is the point of $\Pi$ to which $A_{i} / B_{i}$ belongs and $l_{i}$ is the line of $\Pi$ to which $C_{i} / D_{i}$ belongs. Conversely, to every loop in $\Pi$, there corresponds at least one loop in $A^{*}$. We define $\bar{c}_{1} \sim \bar{c}_{2}$ if $c_{1}$ and $c_{2}$ belong to the same coset of $H^{*(2)}$ in $H^{*}$. Then 
these classes of equivalent loops form a group $\bar{H} \simeq H^{*} / H^{*(2)}$. We define the length of the loop $\bar{c}=\left(P_{1} \stackrel{l_{1}}{\rightarrow} P_{2} \stackrel{l_{2}}{\rightarrow} \cdots \stackrel{l_{n}}{\rightarrow} P_{1}\right)$ to be $n$, the length of an equivalence class to be the length of its shortest member and define $\bar{H}^{(n)}$ to be the subgroup of $\bar{H}$ generated by the classes of length less than or equal to $n$. We can work with the loops in $\Pi$ in the same way as with loops in $A$, $A^{*}$. We observe that replacing in $\bar{c}$ the line $l_{i}$ passing through the points $P_{i}$ and $P_{i+1}$ by any other such line $l_{i}^{\prime}$ does not change the equivalence class of $\bar{c}$.

LEMMA 10.3. $\bar{H}=\bar{H}^{(3)}$.

Proof. Suppose $\bar{c}=\left(P_{1} \rightarrow P_{2} \rightarrow \cdots \rightarrow P_{n} \rightarrow P_{1}\right)$ is an irreducible loop in $\Pi$ of length greater than 3 . If there is a line through $P_{1}, P_{3}$, then

$$
\bar{c}=\left(P_{1} \rightarrow P_{2} \rightarrow P_{3} \rightarrow P_{1}\right)\left(P_{1} \rightarrow P_{3} \rightarrow \cdots \rightarrow P_{1}\right)
$$

which is a product of a loop of length 3 and a loop of length $n-1$, contrary to the assumption that $\bar{c}$ is irreducible. Thus if $a_{i}$ is a coordinate vector for $P_{i}$, either $a_{3}=\alpha p a_{1}$ or $a_{1}=\alpha p a_{3}$ for some $\alpha$. If also there is no line through $P_{1}$ and $P_{4}$, then either $a_{4}=\beta p a_{1}$ or $a_{1}=\beta p a_{4}$ for some $\beta$. Since $P_{1}, P_{3}$ and $P_{4}$ are distinct points, in each of these four cases we must have either $a_{3}=\gamma p a_{4}$ or $a_{4}=\gamma p a_{3}$ for some $\gamma$, contrary to the existence of a line through $P_{3}$ and $P_{4}$. Therefore.

$$
\bar{c}=\left(P_{1} \rightarrow P_{2} \rightarrow P_{3} \rightarrow P_{4} \rightarrow P_{1}\right)\left(P_{1} \rightarrow P_{4} \rightarrow \cdots \rightarrow P_{1}\right),
$$

a product of a loop of length 4 and a loop of length $n-2$. Hence no loop of length greater than 4 is irreducible.

Let $\bar{c}=\left(P_{1} \rightarrow P_{2} \rightarrow P_{3} \rightarrow P_{4} \rightarrow P_{1}\right)$ be any loop of length 4, and let the coordinates of $P_{i}$ be $a_{i}$. Either $\bar{c}$ is reducible or $P_{1}$ and $P_{3}$ are not collinear, and $P_{2}$ and $P_{4}$ are not collinear. Without loss of generality, we may suppose $a_{3}=\alpha p a_{1}$ and $a_{4}=\beta p a_{2}$. Since $P_{1} \neq P_{2}, a_{1}+a_{2} \neq 0$. Put $\boldsymbol{b}=\boldsymbol{a}_{1}+\boldsymbol{a}_{2}$. Then the point $Q$ with coordinates $\boldsymbol{b}$ is collinear with each of the $P_{i}$. Therefore

$$
\begin{aligned}
\bar{c}= & \left(Q \rightarrow P_{1} \rightarrow P_{2} \rightarrow Q\right)\left(Q \rightarrow P_{2} \rightarrow P_{3} \rightarrow Q\right) \\
& \left(Q \rightarrow P_{3} \rightarrow P_{4} \rightarrow Q\right)\left(Q \rightarrow P_{4} \rightarrow P_{1} \rightarrow Q\right) .
\end{aligned}
$$

Therefore every loop of length 4 is reducible and $\bar{H}=\bar{H}^{(3)}$.

THEOREM 4. Suppose $G$ is an abelian p-group of type $\left(\lambda_{1}, \cdots, \lambda_{k}\right)$, $\lambda_{1} \geqq \lambda_{2} \geqq \cdots \geqq \lambda_{k}$, and let $\Lambda$ be a class of projective cyclic quotients of order $p^{\lambda}$. If

(1) $\lambda_{2}<\lambda$, or

(2) $\lambda_{3} \geqq \lambda$, or

(3) $\lambda_{2}=\lambda$ and $p \neq 2$,

then $H(\Lambda)=H^{(2)}(\Lambda)$ and $H\left(\Lambda^{*}\right)=H^{(2)}\left(\Lambda^{*}\right)$. 
Proof. $H(\Lambda) / H^{(2)}(\Lambda) \simeq H\left(\Lambda^{*}\right) / H^{(2)}\left(\Lambda^{*}\right) \simeq \bar{H} . \bar{H}^{(2)}$ is trivial. If $\lambda_{2}<\lambda$, then there is only one point and one line in $\Pi$ and the result follows. For the cases (2), (3), we prove $\bar{H}=\bar{H}^{(2)}$ by showing that every loop of length 3 in $\Pi$ is reducible. The loop $\left(P_{1} \rightarrow P_{2} \rightarrow P_{3} \rightarrow P_{1}\right)$ is reducible if there is a line passing through all three points. If $a_{i}=\left(a_{i 1}, a_{i 2}, \cdots, a_{i r}\right)$ is the coordinate vector of $P_{i}$, then to find such a line, we must find a row vector $x$ such that

$$
\begin{aligned}
& a_{1} \cdot x=\alpha_{1} p^{\mu-1} \\
& a_{2} \cdot x=\alpha_{2} p^{\mu-1} \\
& a_{3} \cdot x=\alpha_{3} p^{\mu-1}
\end{aligned}
$$

with $\alpha_{1}, \alpha_{2}, \alpha_{3} \neq 0 \bmod p$, that is, we must find $\left(x_{1}, \cdots, x_{r}\right)$ such that

$$
\sum_{j} a_{i j} x_{j} p^{\lambda_{1}-\lambda_{j}}=\alpha_{i} p^{\mu-1} \quad\left(\bmod p^{\mu}\right) \text {. }
$$

Put $b_{i j}=a_{i j} p^{\lambda_{1}-\lambda_{j}}, B=\left(b_{i j}\right)$. Then $B$ is a matrix over the ring $Z_{p j}$ of integers $\bmod p^{\mu}$. If

$$
X=\left(\begin{array}{c}
x_{1} \\
\vdots \\
x_{r}
\end{array}\right) \text { and } k=p^{\mu-1}\left(\begin{array}{c}
\alpha_{1} \\
\alpha_{2} \\
\alpha_{3}
\end{array}\right) \text {, }
$$

then the equations are $B \boldsymbol{X}=\boldsymbol{k}$ where $B, \boldsymbol{X}, \boldsymbol{k}$ are matrices over $Z_{p \mu}$.

We define the degree of the residue $x \bmod p^{\mu}$ to be the index of the highest power of $p$ which divides $x$ if $x \neq 0 \bmod p^{\mu}$, and put degree $x=\mu$ if $x \equiv 0 \bmod p^{\mu}$. There exist matrices $P, Q$ over $Z_{p \mu}$ with inverses $P^{-1}$, $Q^{-1}$ over $Z_{p \mu}$ such that

$$
P B Q=\left(\begin{array}{lll|l}
u & 0 & 0 & \\
0 & v & 0 & 0 \\
0 & 0 & w &
\end{array}\right)
$$

where degree $u \leqq$ degree $v \leqq$ degree $w$. Put $\boldsymbol{Y}=Q^{-1} X$. Then the equations $B X=k$ have a solution if and only if the equations $P B Q Y=P K$ have a solution. These equations have the form

where

$$
\begin{aligned}
& u y_{1}=\theta \\
& v y_{2}=\phi \\
& w y_{3}=\psi
\end{aligned}
$$

$$
\boldsymbol{Y}=\left(\begin{array}{c}
y_{1} \\
\vdots \\
y_{r}
\end{array}\right) \text { and }\left(\begin{array}{l}
\theta \\
\phi \\
\psi
\end{array}\right)=P k
$$

whence $\theta, \phi, \psi$ have degree $\geqq \mu-1$. Thus the equations certainly have a 
solution for all $\alpha_{1}, \alpha_{2}, \alpha_{3}$ if $p^{\mu}$ does not divide $w$. But $p^{\mu}$ divides $w$ if and only if there exist $\alpha, \beta, \gamma$ not all $\equiv 0 \bmod p$ such that

$$
\alpha a_{1}+\beta a_{2}+\gamma a_{3}=0 \text {. }
$$

Thus there exists a solution unless for some numbering of $P_{1}, P_{2}, P_{3}$, we have $a_{3}=\alpha a_{1}+\beta a_{2}$. Suppose $\boldsymbol{a}_{3}=\alpha a_{1}+\beta a_{2}$.

Case $1: r \geqq 3$. If we can find a point $Q$ such that each of the sets $P_{1}, P_{2}, Q ; P_{1}, P_{3}, Q ; P_{2}, P_{3}, Q$ is a set of three collinear points, then

$$
\begin{aligned}
\left(P_{1} \rightarrow P_{2} \rightarrow P_{3} \rightarrow P_{1}\right)= & \left(Q \rightarrow P_{1} \rightarrow P_{2} \rightarrow Q\right) \\
& \left(Q \rightarrow P_{2} \rightarrow P_{3} \rightarrow Q\right)\left(Q \rightarrow P_{3} \rightarrow P_{1} \rightarrow Q\right),
\end{aligned}
$$

and each of the loops on the right is reducible. Since $r \geqq 3$, the additive group of row vectors is an abelian group with at least three generators. Therefore there exists a non-trivial cyclic subgroup $\langle\boldsymbol{b}\rangle$ such that $\langle b\rangle \cap\left\langle a_{1}, a_{2}\right\rangle=0$. Suppose $\mathbf{u}, v$ are any two of $a_{1}, a_{2}, a_{3}$ and $\theta b+\phi u+$ $\psi v=0$. Then $\theta b \in\langle b\rangle \cap\left\langle a_{1}, a_{2}\right\rangle$ and therefore $\theta b=0, p$ divides $\theta$ and $\phi \boldsymbol{u}+\psi \mathbf{v}=\mathbf{0}$. But $\boldsymbol{u}, \boldsymbol{v}$ correspond to distinct collinear points. Therefore $p$ divides $\phi, \psi$. Therefore the point $Q$ with coordinates $b$ has the required property.

Case 2: $r=2, \lambda_{2}=\lambda, p \neq 2$. Then $a_{1}=\left(\theta_{1}, \theta_{2}\right), a_{2}=\left(\phi_{1}, \phi_{2}\right)$. Here $\theta_{2}$ and $\phi_{2}$ are residues mod $p$. For some numbering of $a_{1}, a_{2}$, we have $\phi_{1}=t \theta_{1}$ for some integer $t$, and $\theta_{1} \neq 0$. Put $b=a_{2}-t a_{1}=\left(0, \phi_{2}-t \theta_{2}\right)$ and let $Q$ be the point with coordinates $b$. Then $Q$ is collinear with each of the points $P_{1}, P_{2}, P_{3}$, and

$$
\begin{aligned}
\left(P_{1} \rightarrow P_{2} \rightarrow P_{3} \rightarrow P_{1}\right)= & \left(Q \rightarrow P_{1} \rightarrow P_{2} \rightarrow Q\right) \\
& \left(Q \rightarrow P_{2} \rightarrow P_{3} \rightarrow Q\right)\left(Q \rightarrow P_{3} \rightarrow P_{1} \rightarrow Q\right) .
\end{aligned}
$$

It is sufficient to prove each of these three loops reducible.

If $\boldsymbol{u}, \boldsymbol{v}$ are any two of $\boldsymbol{a}_{1}, \boldsymbol{a}_{2}, \boldsymbol{a}_{3}$, then in each case, one of the three vectors $\boldsymbol{a}, \boldsymbol{v}, \boldsymbol{b}$ is expressible in terms of the other two, as is obvious if $u, v$ are $a_{1}, a_{2}$ or $a_{1}, a_{3}$. For $u=a_{2}, v=a_{3}=\alpha a_{1}+\beta a_{2}$, we have either $\alpha=r t$ for some $r$, in which case $a_{3}=(r+\beta) a_{2}-r b$, or $t=s \alpha$ for some $s$, in which case $b=(1+s \beta) a_{2}-s a_{3}$. Thus in each case, $\theta u+\phi v+\psi b=0$ for some $\theta, \phi, \psi$ not all congruent $0 \bmod p$. But $p b=0$. Further, $\theta u+\phi v=0$ implies $\theta \equiv \phi \equiv 0 \bmod p$ since $u, v$ correspond to distinct collinear points. Hence $\psi \not \neq 0 \bmod p$. But $p(0,1)=\mathbf{0}$ and so if $\theta \equiv \phi \equiv 0 \bmod p$, then $-\psi b$ has second component 0 and so $\psi \boldsymbol{b}=\mathbf{0}$. Hence $\theta, \phi$ are not both congruent $0 \bmod p$ and we may suppose $v=\theta u+\psi b, \psi \neq 0 \bmod p$. We have thus reduced the problem to the case $a_{3}=\alpha a_{1}+a_{2}, a_{2}=(0,1)$.

For $x=(x, 1)$, we have $a_{2} \cdot x=p^{\mu-1}$. Since $a_{1}=\left(\theta_{1}, \theta_{2}\right)$ with $\theta_{1} \neq 0$, we can choose $x$ such that 
Then

$$
a_{1} \cdot x=\left\{\begin{aligned}
p^{\mu-1} & \text { if } \alpha \equiv 0 \bmod p \\
\beta p^{\mu-1} & \text { where } \alpha \beta \equiv 1 \bmod p \text { if } \alpha \neq 0 \bmod p .
\end{aligned}\right.
$$

$$
a_{3} \cdot x=\left\{\begin{aligned}
p^{\mu-1} & \text { if } \alpha \equiv 0 \bmod p \\
2 p^{\mu-1} & \text { if } \alpha \neq 0 \bmod p .
\end{aligned}\right.
$$

Since $p \neq 2$, the line with coordinates $x$ passes through $P_{1}, P_{2}, P_{3}$ and the loop $\left(P_{1} \rightarrow P_{2} \rightarrow P_{3} \rightarrow P_{1}\right)$ is reducible.

\section{The determination of $\pi(\Lambda)$}

TheOREM 5. Let $G$ be an abelian p-group of type $\left(\lambda_{1}, \lambda_{2}, \cdots, \lambda_{k}\right)$, $\lambda_{1} \geqq \lambda_{2} \geqq \cdots \geqq \lambda_{k}$, and let $\Lambda$ be a class of projective cyclic quotients of order $p^{\lambda}$. If $\lambda_{2} \geqq \lambda$, then $\pi(\Lambda)$ is the group of all automorphisms of the cyclic group of order $p^{\lambda}$. If $\lambda_{2}<\lambda$, then $\pi(\Lambda)$ is the group of all automorphisms of the group $\langle x\rangle$ of order $p^{\lambda}$ of the form

$$
x \rightarrow x^{1+t p^{\lambda-\lambda_{2}}} \quad(t \text { integer }) \text {. }
$$

Proof. (1) We consider first the case $G=\langle x, y\rangle, x^{p \lambda}=y^{p \mu}=1$, $\mu \leqq \lambda$. By Theorem $4(1), H^{*}=H^{*(2)}$. Let $c=(A \rightarrow G / B \rightarrow C \rightarrow G \mid D \rightarrow A)$ be any loop of length 4 in $A^{*}$. Without loss of generality, we may take $A=\langle x\rangle, B=\langle y\rangle$. Since $C \cap B=1, C^{p^{2-1}} B$ and therefore $C=\left\langle x y^{r}\right\rangle$ for some $r$. Since $|D|=p^{\mu}$ and $D^{p^{\mu-1}} \backslash A, D=\left\langle x^{s p^{*}} y\right\rangle$ for some $s$, where $e=\lambda-\mu$. If $e \geqq 1$, then for any values $r$, s, we have $\left\langle x y^{r}\right\rangle \cap\left\langle x^{s p^{4}} y\right\rangle=1$ and there is a loop with these values of $r$, s. If $e=0$, then $\left\langle x y^{r}\right\rangle \cap\left\langle x^{s} y\right\rangle=1$ if and only if $r s \neq 1 \bmod p$ and for any values of $r, s$ such that $r s \neq 1 \bmod p$, there is a corresponding loop. In this loop $c$,

$$
\begin{aligned}
& \langle x\rangle \rightarrow G /\langle y\rangle \rightarrow\left\langle x y^{r}\right\rangle \rightarrow G /\left\langle x^{s p^{*}} y\right\rangle \rightarrow\langle x\rangle, \\
& x \rightarrow\left\{x y^{t} \mid \text { all } t\right\} \rightarrow x y^{r} \rightarrow\left\{x^{1+t s p^{*}} y^{r+t} \mid \text { all } t\right\} \rightarrow x^{1-r s p^{*}} .
\end{aligned}
$$

Thus $x \alpha(c)=x^{1-r s p^{*}}$. If $e=0$, then every automorphism of $\langle x\rangle$ is given by some values of $r$, s. If $e \geqq 1$, then every automorphism of the form $x \rightarrow x^{1+t p^{*}}$ is given by some values of $r, s$, and since these automorphisms form a group, $\pi(\Lambda)$ contains only these automorphisms.

(2) If $\lambda_{2} \geqq \lambda$, then by (1), all automorphisms of the cyclic group of order $p^{\lambda}$ are given by loops of length 4 within $\Omega_{\lambda}(G)$, and therefore $\pi(\Lambda)$ is the group of all automorphisms of the cyclic group of order $p^{\lambda}$.

(3) Suppose $\lambda_{2}<\lambda$. Since $\pi(\Lambda)$ does not depend on the class $A$, we need only consider the class $\Lambda$ contained in $\Omega_{\lambda}(G)$. Hence we may suppose $\lambda_{1}=\lambda$. By Theorem $4(1), H(\Lambda)=H^{(2)}(\Lambda)$ and it is sufficient to find $\alpha(c)$ for all loops $c$ of length 4 . If $A / B$ is a pit, then $B=1$. Let 


$$
c=(A \rightarrow G / B \rightarrow C \rightarrow G / D \rightarrow A)
$$

be any loop of length 4 in $A^{*}$. Then $c$ is homotopic (in $A$ ) to

$$
A \rightarrow(A \cup C) /(A \cup C) \cap B \rightarrow C \rightarrow(A \cup C) /(A \cup C) \cap D \rightarrow A .
$$

Thus to determine the $\alpha(c)$ given by loops of length 4 in $\Lambda$, it is sufficient to determine the $\alpha(c)$ given by loops of length 4 within the two-generator subgroups of $G$. Since every two-generator subgroup of exponent $p^{\lambda}$ of $G$ is isomorphic to a subgroup of the group of type $\left(\lambda, \lambda_{2}\right)$, the result follows from (1).

\section{References}

[1] Barnes, D. W., Lattice embeddings of prime power groups, This Journal 2 (1961) 17-34.

[2] Tamaschke, O., Die Kongruenzrelationen im Verband der zugänglichen Subnormalteiler. Math. Zeitschr. 75 (1961) 115-126.

[3] Seifert, H. and Threlfall, W., Lehrbuch der Topologie. New York, 1947.

[4] Zassenhaus, H., The theory of groups. Second English edition. New York, 1058.

University of Sydney. 